\title{
Adaptive Signal Decomposition Based on Local Narrow Band Signals
}

\author{
Silong Peng and Wen-Liang Hwang ${ }^{\dagger}$ \\ Institute of Automation, The Chinese Academy of Sciences, Beijing, China \\ Institute of Information Science, Academia Sinica, Taiwan ${ }^{\dagger}$
}

December 22, 2007

\begin{abstract}
We propose an operator-based method of adaptive signal decomposition, whereby a local narrow band signal is defined in the null space of a singular local linear operator. Based on the definition and the algorithm, we propose two types of local narrow band signals and two singular operator estimation methods for adaptive signal decomposition. We show that our approach can solve a special case of Huang et al.'s empirical mode decomposition algorithm. For signals that cannot be resolved by our method or the empirical mode decomposition algorithm, we propose a hybrid approach. Conceptually, the approach applies the empirical mode decomposition algorithm, followed by our algorithms. Our experiments show that the proposed hybrid approach can solve a wide range of complex signals effectively.
\end{abstract}

\section{Introduction}

In recent years, the local narrow band signal (or "time-varying" narrow band signal) has attracted a great deal of attention in the field of signal and image processing $[1,2,3,4,5,12,13]$. A singular local linear operator is a linear operator whose support is compact and whose null space is not zero. We provide a local narrow band signal model in which the signals are vanished by a singular local linear operator. In other words, the local narrow band signals are in the null space of the singular 
local linear operator. Thus, we can use the operator to extract local narrow band signals from its null space. To do this, we apply a singular local linear operator to a signal in order to extract the local narrow band components of the signal for signal decomposition purposes. Signal decomposition has been widely applied in many fields. The most widely used approach models a signal as a superposition of basic signals. For example, the basic component of Huang et al.'s empirical mode decomposition (EMD) algorithm is the intrinsic mode function (IMF) [9]. We have developed a signal model in which the signal consists of local narrow band signals. Of particular interest is our approach's ability to successfully apply a decomposition algorithm to extract local narrow band signals. As we will show, our algorithm is adaptive to a signal and can be used to solve an optimization problem in which the solution is constrained. Specifically, in each decomposition, we obtain a local narrow band signal in the null space of a singular local linear operator, which is estimated from the extremal points of the signal.

The EMD algorithm models a signal as the superposition of IMFs, each of which is obtained by a sifting process. We show that if the positions of the extremal points of a signal are invariant during the sifting process, then the IMF is in the null space of a singular local linear operator. In this case, the EMD algorithm and our approach share a common conceptual framework; thus, the proposed signal decomposition algorithm can be used to obtain the IMF.

We propose two singular local linear operators whose null spaces are of particular interest; one is based on integration and the other on differentiation. We also construct a signal that can be correctly decomposed into a superposition of local narrow band signals by the EMD algorithm, but not by the proposed singular local linear operators. Although it has been shown empirically that the EMD algorithm is very effective in decomposing a signal $[14,11,6,18]$, the solution of the algorithm is in a larger class of signals than the local narrow band signals. We thus propose a hybrid framework that uses the EMD algorithm to retrieve a signal, after which our proposed differentiation-based algorithm is applied to extract a local narrow band signal.

The remainder of this paper is organized as follows. In Section 2, we define a singular local linear operator and a local narrow band signal. We also propose two singular local linear operators: one based on an integral operator, and the other on a differential operator. In Section 3, we introduce our adaptive signal decomposition 
algorithms for estimating the singular operators and extracting local narrow band signals. In addition, we analyze a special case of the EMD algorithm and show that the IMF of a signal in this case can be obtained by our approach. In Section 4, we present our hybrid approach, which conceptually combines the EMD algorithm and our approach for signal decomposition. In Section 5, we demonstrate the efficacy of applying the proposed methods on two real-world signals. We then present our conclusions in Section 6.

\section{Local Narrow Band Signal}

It is known that a narrow band signal can be expressed as $A(t) \cos (\omega t+\phi(t))$, where $A(t)$ is a band-limited signal whose maximal frequency is much smaller that $\omega$, and $\phi(\mathrm{t})$ is a slow-varying phase function. Hence, the narrow band signal can be extended to a local narrow band signal. A signal is a local narrow band if, at any point, there exists a neighborhood interval such that the signal can be approximated as a narrow band signal on the interval. For a narrow band signal $(a t+b) \cos (\varpi t+c)$ with a neighborhood interval of $t$, we have

$$
\left(\frac{1}{\varpi^{2}} \frac{d^{2}}{d t^{2}}+1\right)^{2}(a t+b) \cos (\varpi t+c)=0 .
$$

The narrow band signal $(a t+b) \cos (\varpi t+c)$ is in the null space of the differential operator $\left(\frac{1}{\varpi^{2}} \frac{d^{2}}{d t^{2}}+1\right)^{2}$. We can use this perspective to define local narrow band signals.

Definition 1. A linear operator $T$ from $L_{2}(R)$ to $L_{2}(R)$ is called a local linear operator if, for each $t \in R$, there exists a neighbor $B_{t}$ of $t$ such that

$$
T(S)(t)=T\left(\left.S\right|_{B_{t}}\right)(t)
$$

where $\left.S\right|_{B_{t}}(t)=S(t)$ when $t \in B_{t}$, and 0 otherwise. If $T$ is a singular operator, we call $T$ a singular local linear operator.

From the definition, a local linear operator has a neighborhood, $B_{t}$. Note that each neighborhood has a different operator. We present two types of singular local linear operator because their local narrow band signals have generated a great deal of interest in signal processing applications. We define the first type as

$$
T(S)(t)=\int_{B_{t}} S(x) d x
$$


The local narrow signal of the operator is defined as follows.

Definition 2. A local narrow band signal $S(t)$ is called the first type of local narrow band signal of order $n$ if there exists a singular local linear operator, as in (2), and a number $n$ such that

$$
T^{n}(S)(t)=0 \text {, a.e. }
$$

The local narrow band signal in (2) with $n=1$ is defined in $[1,2]$. In fact, we often find signals of the form $P_{n}(t) \cos (\varpi t+c)$. For such signals, using the first order integral is not sufficient to make the integral zero in any period. Therefore, we extend the definition in $[1,2]$ to a higher order integral with $n \geq 1$.

The first type of local narrow band signal is defined according to the integral operator. However, we can also define a local narrow band signal by using a differential operator. Let us define the operator as follows:

$$
T_{\alpha}=\sum_{k \in Z} \alpha(k) \frac{d^{k}}{d t^{k}},
$$

where $\{\alpha(k)\}$ is a square summable sequence belonging to $l_{2}(Z)$. This operator is a singular operator with many eigenfunctions corresponding to eigenvalue 0. Here, we give a derivation of the eigenfunctions. Let $A(x)=\sum_{k \in Z} \alpha(k) x^{k}$, and let $\Omega$ be the set of zeros of $A(x)$. For any $m$ elements $\left\{\lambda_{k}, k=1, \cdots, m\right\}$ in $\Omega, f_{m}(t)=\sum_{k=1}^{m} c_{k} e^{\lambda_{k} t}$ is an eigenfunction of $T_{\alpha}$ corresponding to eigenvalue 0 , where $\lambda_{k}$ can be a real or a complex number. For a signal $P_{n}(t) \cos (\varpi t+c), \lambda_{k}$ is an imaginary number. The local narrow band signal of the operator (4) is given by the following definition.

Definition 3. A local narrow band signal $S(t)$ is called the second type of local narrow band signal if for each point $t$, there exists a sequence $\alpha \in l_{2}(Z)$, and all the zeros of $A(x)=\sum_{k \in Z} \alpha(k) x^{k}$ are imaginary numbers such that

$$
T_{\alpha}(S)(t)=0 \text {, a.e. }
$$

The following example illustrates that a local harmonic function is in the null space of a differential operator.

Example 1. Let

$$
T_{n}=\left(\frac{1}{\varpi^{2}} \frac{d^{2}}{d t^{2}}+1\right)^{n}
$$


The solution of $T_{n}(S)(t)=0$ is

$$
S(t)=P_{n}(t) \cos (\varpi t+c)
$$

where $P_{n}(t)$ is a piecewise polynomial of degree $n-1$.

\section{Singular Operator Signal Decomposition}

The main purpose of signal decomposition is to decompose a signal into a superposition of signals that have certain features. Since signal decomposition problems are usually ill-posed, we propose regularization methods that decompose a signal into a superposition of local narrow band signals. Specifically, given a signal $S(t)$, we seek some local narrow band signals $S_{k}(t)$ and a residual $P(t)$ such that

$$
S(t)=\sum_{k} S_{k}(t)+P(t)
$$

To solve (8), we propose an adaptive signal decomposition technique that uses an optimization method to find a component, $S_{k}$, at each iteration. Consider the following problem: given a signal $S(t)$, find $S_{1}(t)$ and $U(t)$ such that

$$
S(t)=S_{1}(t)+U(t)
$$

where $S_{1}(t)$ is either the first or the second type of local narrow band signal, and $U(t)$ is the residual. Since $S_{1}(t)$ is a local narrow band signal, there exists a singular linear operator $\mathcal{T}$ such that

$$
\mathcal{T}\left(S_{1}\right)(t)=0, \text { a.e. }
$$

To obtain $U(t)$, we solve the following optimal problem:

$$
\tilde{U}(t)=\arg \min \left\{\|\mathcal{T}(S-U)\|^{2}+\lambda\|D(U)\|^{2}\right\}
$$

where $D$ is an operator that regulates $U$. Minimizing the term $\|\mathcal{T}(S-U)\|^{2}$ indicates that $S-U$ is a local narrow band signal with respect to the operator $\mathcal{T}$. The analytical solution of (11) is

$$
\tilde{U}=\left(\mathcal{T}^{*} \mathcal{T}+\lambda D^{*} D\right)^{-1} \mathcal{T}^{*} \mathcal{T} S
$$


The procedure decomposes a signal into the local narrow band signal $S_{1}=S-\tilde{U}$ and the residual $\tilde{U}$, which becomes the signal at the next iteration. In the following subsections, we analyze when a signal is decomposed into a superposition of the first or second type of local narrow band signal.

\subsection{First Type of Local Narrow Band Signal Decomposition}

Given a signal $S$, to find $\tilde{U}$ with respect to the integral operator (2), we need to determine the neighborhood $B_{t}$ at each point $t$. In fact, if $B_{t}$ is deduced from the periodic function with a zero mean, such as $\cos (t)$, it is the local period of $S-U$ at point $t$.

Assume that $S(t)$ is a compactly supported signal with support $[a, b]$. Let $L_{0}=$ $\left\{p_{0}<p_{1} \cdots<p_{n-1}<p_{n}\right\}$ be the set of points where $S(t)$ achieves the local maximal values, and let $L_{1}=\left\{q_{0}<q_{1} \cdots<q_{m-1}<q_{m}\right\}$ be the set of points where $S(t)$ achieves the local minimal values. We assume that the discrete extremal points in $L_{0} \cup L_{1}$ are all extremal points of $S(t)$. Except for boundary points, each point in $L_{0}$ must lie between two neighbors in $L_{1}$. We make a similar assumption about the points in $L_{1}$. The points in $L_{0}$ and $L_{1}$ split the interval $[a, b]$ into several smaller intervals $I_{i}$, each of which has one bound in $L_{0}$ and the other bound in $L_{1}$; that is, $[a, b]=\cup I_{i}$ and $I_{i} \cap I_{i+1}=p_{i}$ or $q_{i}$. We then define

$$
B_{t}=\left[t-\delta_{t}, t+\delta_{t}\right]
$$

where

$$
\delta_{t}=\left(\left|I_{i}\right|+\left(\left|I_{i-1}\right|+\left|I_{i+1}\right|\right) / 2\right) / 2 \text { for } t \text { lies in } I_{i} .
$$

The estimation of $B_{t}$ is valid for an inside interval of $[a, b]$, but not for an endinterval. Because we do not have any information about internals outside $[a, b]$, we use a prediction method to determine the interval at the boundary. For the left end-interval of $[a, b]$, we let

$$
B_{t}=[a, a+\eta] \text { for } t \in[a, a+\eta]
$$

where

$$
\eta=\left(\left|I_{2}\right|+\left|I_{3}\right|\right) / 2
$$


The right end-interval can be similarly defined. From $B_{t}$, we have an integral operator of the form in (2). In a discrete case, we use the average, instead of the integral, in the computation. The input signal is a column vector $S$. The local integral operator is approximated as a matrix $A$, where each row in $A$ corresponds to a local average with a window, determined from a local period of extremal points in $S$. To solve (12), we must choose the operator $D$, which is defined as a derivative operator, as in many regularization approaches. Therefore, $\tilde{U}$ is a smooth component of $S$.

The decomposition (9) must be performed many times until the norm of the output is zero. We thus obtain a series of outputs $U_{i}$, with $i=1, \ldots, N-1$. Let us set $U_{0}=S$, and $U_{N}=0$; then the signal $S$ can be written as the superposition of the first type of local narrow band signal:

$$
S=\sum_{i=1}^{N} S_{i}(t), \text { where } S_{i}(t)=U_{i-1}-U_{i} .
$$

The step-by-step algorithm for signal decomposition is as follows.

\section{Algorithm I:}

Step 1. Set $U_{0}=S$ and $i=1$.

Step 2. Determine $B_{t}$ at each point $t$.

Step 3. Construct the average matrix $\mathrm{A}_{i}$ and the difference matrix $\mathrm{D}_{i}$.

Step 4. Choose $\lambda_{i}$ and solve the equation

$$
U_{i}=\left(A_{i}^{T} A_{i}+\lambda_{i} D_{i}^{T} D_{i}\right)^{-1} A_{i}^{T} A_{i} U_{i-1}
$$

Step 5. If $\left\|U_{i}\right\|$ is smaller than a given threshold, the algorithm is terminated; otherwise, set $i=i+1$, and go to Step 2 .

The following example illustrates the signal decomposition process.

Example 2. Decompose $S(t)=\sin (2 t)+(\sin (t)+2) \sin (10 t)$ by Algorithm I. We choose $n=2$ in (3), which corresponds to choosing the assumption that $S(t)$ can be locally approximated as $\left(c_{1} t+c_{2}\right) \cos \left(\varpi t+c_{3}\right)$. The decomposition results are shown in Figure 1. 

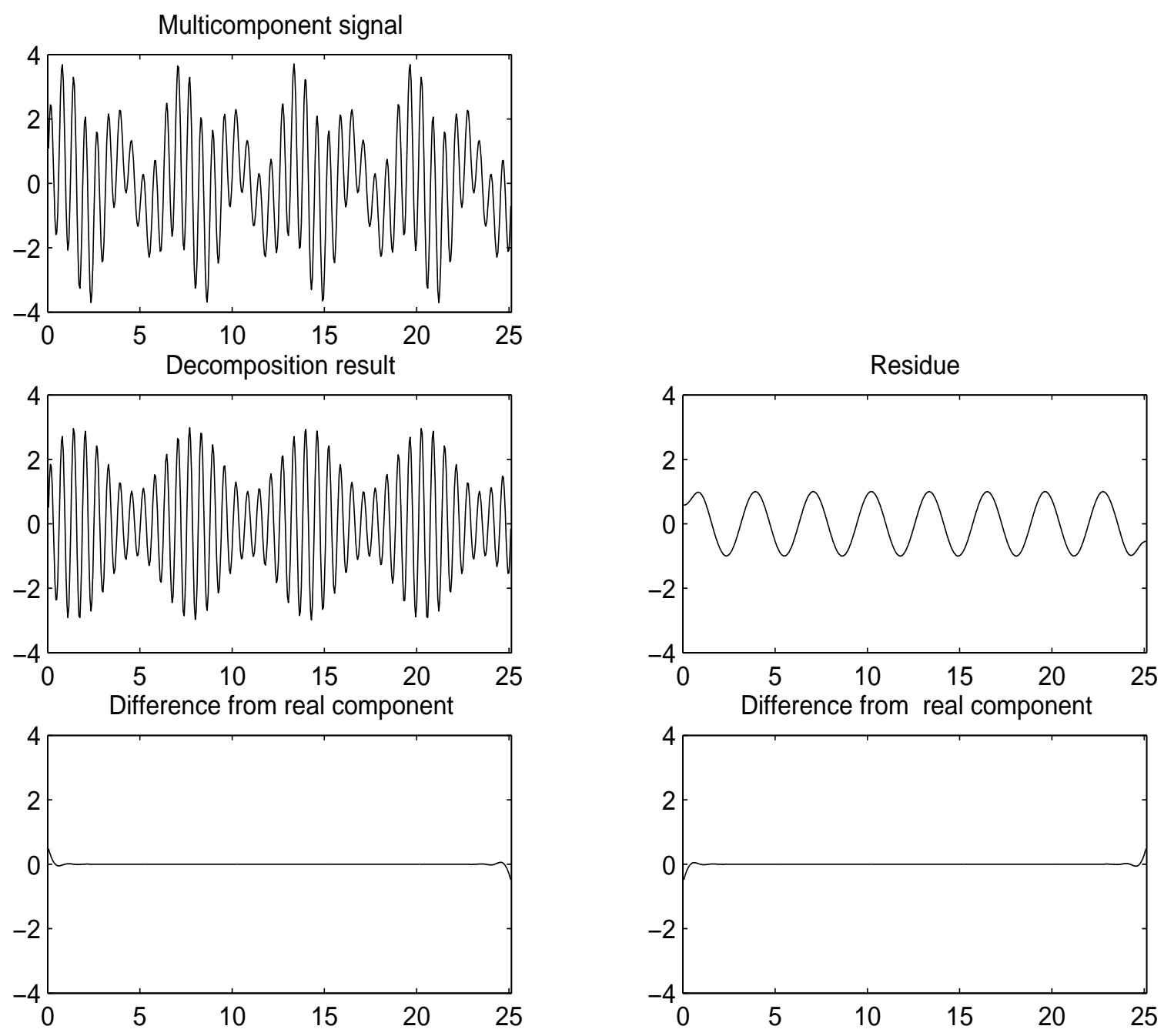

Figure 1: Top left: Signal $S(t)=\sin (2 t)+(\sin (t)+2) \sin (10 t)$. The extracted component is shown in the middle left subfigure, while the residue is in the middle right subfigure. Bottom Left: the difference between the extracted component in the middle left subfigure and $(\sin (t)+2) \sin (10 t)$. Bottom Right: the difference between the residue in the middle right subfigure and $\sin (2 t)$. Except for the boundary distortion, the maximal difference of the bottom two signals is less than 0.005. The parameter $\lambda$ is set at 100 . 


\subsection{Second Type of Local Narrow Band Signal Decomposi- tion}

To decompose a signal into a superposition of the second type of local narrow band signal, we need to determine the operator in (4). In the following, we focus on the operator of the form given in (6) with $n=2$ :

$$
T_{2}=\left(\frac{1}{\varpi^{2}} \frac{d^{2}}{d t^{2}}+1\right)^{2} .
$$

To characterize this operator, we only need to estimate the local frequencies, but it is very difficult to do this without knowing the exact signal. However, if we assume that the discrete extremal points are continuous and no other extremal points exist, we can estimate the phase function $\phi(t)$ from the positions of the extremal points. The local frequencies can then be obtained by taking the derivative of the phase function. Let $\left\{r_{0}<r_{1} \cdots<r_{n-1}<r_{n}\right\}$ be the positions of the local extremal points of $S(t)$. We assume that the phase of $S(t)$ varies smoothly, and use a local polynomial function to fit the extremal points to obtain the estimated phase function $\phi(t)$. The constraint on $\phi(t)$ is $\phi\left(r_{k}\right)=k \pi+b$, where $b$ is either $\pi$ or 0 , based on the assumption that if $r_{k}$ is a local maximum, then $\phi\left(r_{k}\right)=2 \pi l$, where $l$ is a natural number. We now present the algorithm that decomposes a signal into a superposition of the second type of local narrow band signal with respect to the singular operator in (19).

\section{Algorithm II:}

Step 1. Set $U_{0}=S, i=1$.

Step 2. Find the local extremal points.

Step 3. Determine the value of $b$ from the phase of a local maximum that satisfies $k \pi+b=2 \pi l$, where $l$ is a natural number.

Step 4. Use interpolation to obtain the phase function $\phi(t)$ under the condition that $\phi\left(r_{k}\right)=k \pi+b$. The derivative of the phase function at any point $t_{j}$ is the local frequency $\omega_{j}$ at that point.

Step 5. Construct the matrix $A_{i}$ as

$$
A_{i}=\left(\Omega_{i} D+I\right)^{2} \text {, }
$$


where $\Omega_{i}$ is a diagonal matrix, $\Omega_{i}(j, j)=1 / \varpi_{j}^{2}, D$ is the discrete second order derivative matrix, and $I$ is the identity matrix.

Step 6. Use (12) to obtain $U_{i}$ as follows:

$$
U_{i}=\left(A_{i}^{T} A_{i}+\lambda_{i} I\right)^{-1} A_{i}^{T} A_{i} U_{i-1}
$$

Step 7. If $\left\|U_{i}\right\|$ is smaller than a given threshold, the algorithm is terminated; otherwise, set $i=i+1$ and go to Step 2 .

The signal in the following example is the same as that in Example 2. Here, we explain how it is decomposed into a superposition of the second type of local narrow band signal.

Example 3. Given the signal in Example 2, $S(t)=\sin (2 t)+(\sin (t)+2) \sin (10 t)$, we obtain the two-component decomposition results by using the operator in (19), as shown in Figure 2. 

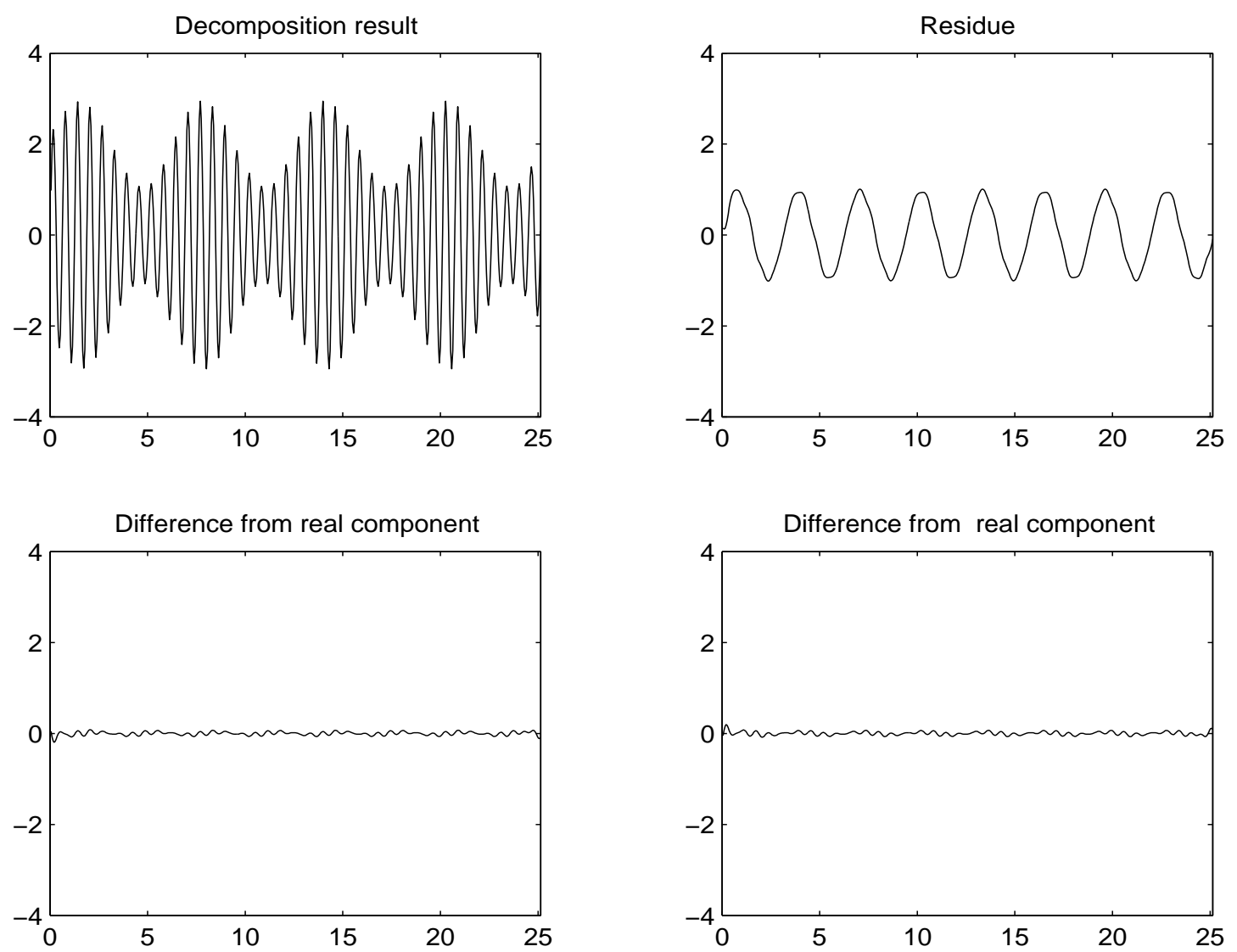

Figure 2: Top left: the extracted component. Top right: the residual signal. Bottom left: the difference between the component extracted in the top left subfigure and $(\sin (t)+2) \sin (10 t)$. Bottom Right: the difference between the residue in the top right subfigure and $\sin (2 t)$. The maximal value of the bottom two signals is less than 0.06 . The parameter $\lambda$ is set at 0.025 . 
The proposed operator-based signal decomposition scheme can be used to extract a signal from the null space of any singular local linear operator. In the next subsection, we show that a special case of EMD decomposition can be formulated as the proposed method and the IMF of the signal satisfying the case can be obtained by our approach.

\subsection{A Special Case of IMF Decomposition}

An IMF must satisfy two conditions: (1) the number of extrema and the number of zero-crossings can differ at most by one; and (2) the mean value of the envelopes defined by the local maxima and the local minima must be zero. The EMD algorithm decomposes a signal into a superposition of IMFs as follows:

$$
S(t)=\sum_{k} C_{k}(t)+P(t)
$$

where $C_{k}(t)$ is an IMF, and $P(t)$ is the residual. An IMF can be obtained by applying the following sifting procedure. Given a signal $s(t)$, the procedure first finds the extremal points, and then computes the mean value $m(t)$ of the envelopes of the extrema. If the mean value is not zero, the procedure is applied to the new signal $s(t)-m(t)$. The sifting process is repeated many times until the mean value of a signal is zero and the signal is an IMF.

We consider a special case of IMF computation in which the positions of the local extrema of a signal are invariant during the sifting process. Therefore, the calculation of the mean value during the sifting process can be represented as a linear operator that does not change at each iteration. The EMD sifting procedure for this case is rewritten as follows:

Step 1. Set $S_{0}=S$, and $i=1$.

Step 2. Find the local extremal points.

Step 3. Construct a matrix $A$ to compute the mean value of the upper and lower envelopes. The matrix $A$ can be written as $\frac{M C+N D}{2}$, where $M$ is an interpolation matrix in which each column is a cardinal interpolating vector; and $C$ is a matrix in which each row has an element of value 1 corresponding to the position of a maximum and 0 in the other elements. $N$ and $D$ are similar to $M$ and $C$ respectively for minima. 
Step 4. Compute $S_{i}=S_{i-1}-A S_{i-1}$.

Step 5. If $\left\|A S_{i}\right\|$ is smaller than a given threshold, terminate the algorithm; otherwise, go to Step 4.

Note that, in Step $\mathbf{5}$, we use $\left\|A S_{i}\right\|$ to determine when to terminate the algorithm. When $\left\|A S_{i}\right\|$ is small enough, according to Step 4, we have $S_{i+1} \approx S_{i}$. Hence, no further iteration is needed. To determine the convergence of $S_{i}$, we study the convergence of $U_{i}=S-S_{i}$. From the procedure, we have

$$
U_{i}=\sum_{k=0}^{i}(I-A)^{k} A S .
$$

Because $A$ is an interpolation matrix, it is a singular linear operator. For a signal $S$, if $(I-A)^{k} A S$ is not zero for all $k$, then $U_{i}$ does not converge. As in the EMD algorithm, it is not clear whether the above procedure is convergent or not.

Note that, for the case under discussion, we can use the optimization method proposed in (11) to extract an IMF, which is in the null space of the singular local linear operator $A$.

\section{Hybrid Signal Decomposition Algorithm}

The proposed adaptive signal decomposition algorithm decomposes a signal into a superposition of component signals in the null space of a singular local linear operator. The efficacy of our method is demonstrated by Examples 3 and 4. However, there are signals that some of operators cannot correctly decompose into a superposition of local narrow band signals, as illustrated by the following two examples.

First, the signal

$$
S(t)=\sin (2 t)-\cos (4 t) / 4-1 / 4
$$

has an interesting property in that its extremal points are the same as those of $\sin (2 t)$. Although the signal is composed of two monotone components, the local average, calculated from the extremal points, is zero. Consequently, the first type of singular operator can not decompose the signal. However, because the correct frequency of $\sin (2 t)$ can be estimated from the extremal points and the differential operator does not vanish the signal, the second type of singular operator can correctly decompose the signal as the superposition of local narrow band signals. 
In the second example, neither the first nor the second type of operator can correctly decompose the following signal into two monotone signals:

$$
S(t)=\sin (2 t)-\cos (4 t) / 3
$$

The interesting property of this signal, shown in Figure 3, is that neither the local frequency of $\sin (2 t)$ nor that of $\cos (4 t) / 3$ can be correctly determined from its extremal points. The second type of singular operator thus uses an operator with an incorrect frequency to extract the component signal, while the first type of singular operator uses an operator with incorrect neighborhoods.

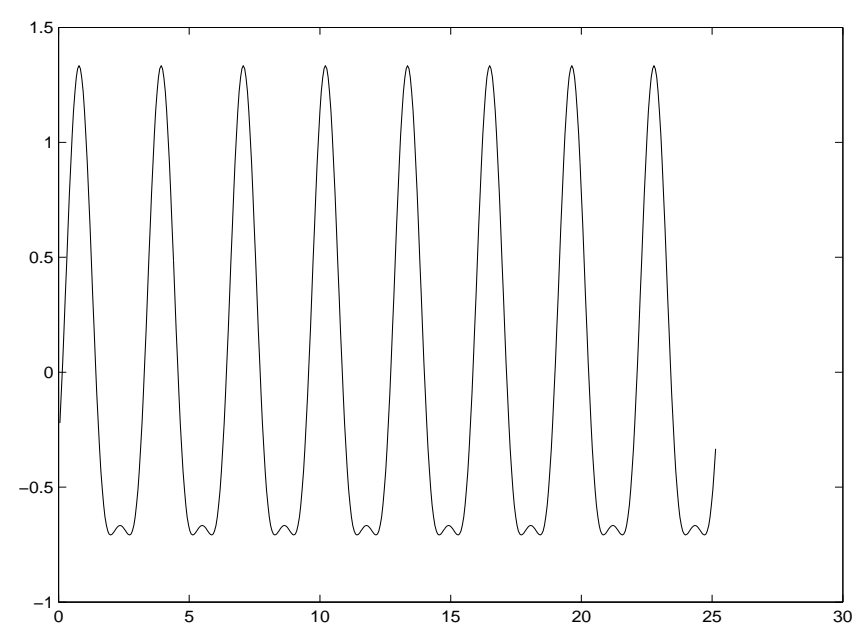

Figure 3: Signal $\sin (2 t)-\cos (4 t) / 3$. The extremal points of the signal are different from those of $\sin (2 t)$ and $\cos (4 t) / 3$.

The EMD algorithm provides another way of performing adaptive signal decomposition. However, the solution of EMD decomposition lies in a larger class of signals that, in general, cannot be characterized by the null spaces of the first and second type of singular local linear operators. For the signals given in (24) and (25), EMD cannot decompose the first signal into two monotone components, but it can successfully decompose the second signal. From the extremal points of the first signal, the EMD algorithm determines that the signal is an IMF and stops the decomposition process. As indicated in [17], the values of $a$ and $f$ determine whether EMD represents a signal of the following form, with $0<f<1$,

$$
\cos (2 \pi t)+a \cos (2 \pi f t+\phi)
$$


as the sum of two separate unmodulated tones, or as a single modulated waveform. The first signal is the case discussed in [17] with $a f^{2}=1$. For the second signal, where the component signals can not be derived directly from the extremal points of the signal, the EMD succeeds in decomposing the signal. The sifting process of EMD varies the extremal points at each iteration and obtains the correct decomposition of the signal after several iterations.

The first and second types of singular operator are constrained by the extremal points of a signal. Although EMD is less constrained by the extremal points, it is not specifically designed to extract local narrow band signals. The IMF is a larger class of oscillatory signals that can not be characterized easily by a singular local linear operator. Thus, we propose the following hybrid approach for local narrow band signal extraction. We use EMD to extract an IMF, which may not be a local narrow band signal, and then use the second type of singular operator to specifically extract a local narrow band signal from the IMF.

However, the convergence of the EMD sifting algorithm is not reported in [9]. Therefore, before we can proceed with our approach, we must modify the EMD algorithm so that it will always converge. Next, we present the modified algorithm.

\section{Modified EMD Sifting Algorithm:}

Step 1. Set $S_{0}=S, i=1$.

Step 2. Find the local extremal points.

Step 3. Construct a matrix $\mathrm{A}_{i}$, which is equivalent to computing the mean envelope.

Step 4. Compute $S_{i}=S_{i-1}-\mu^{i-1} A_{i} S_{i-1}$, where $\mu<1$ is a given number.

Step 5. If $\left\|S_{i}-S_{i-1}\right\|$ is smaller than a given threshold, terminate the algorithm; otherwise, set $i=i+1$ and go to Step 2 .

Note that the only difference between our algorithm and the EMD algorithm is the operation in Step 4, where the multiplier $\mu^{i-1}$ ensures the convergence of our algorithm. The following theorem proves that our algorithm is always convergent.

Theorem: The Modified EMD sifting Algorithm converges when $\mu<1$. 


\section{Proof:}

First, we prove that $\left\|S_{i}\right\|<\infty$. From the iterations, we observe that

$$
S_{i}=\prod_{k=1}^{i}\left(I-\mu^{k-1} A_{k}\right) S .
$$

Because $A_{k}$ is a matrix that performs interpolation and obtains the average of the upper and lower envelopes, its norm is bounded by 1 . Therefore,

$$
\left\|S_{i}\right\| \leq \prod_{k=1}^{i}\left(1+\mu^{k-1}\right)\|S\| .
$$

Moreover because $e^{x} \geq 1+x$ for $x \geq 0$, we have

$$
\left\|S_{i}\right\| \leq \prod_{k=1}^{i}\left(1+\mu^{k-1}\right)\|S\| \leq \prod_{k=1}^{\infty}\left(1+\mu^{k-1}\right)\|S\| \leq e^{\sum_{k=1}^{\infty} \mu^{k-1}}\|S\| .
$$

For a given $\mu<1$, we have $\left\|S_{i}\right\| \leq C=e^{\frac{1}{1-\mu}}\|S\|$. To confirm the convergence of $S_{i}$, we study the convergence of $S-S_{i}$, which yields

$$
S-S_{i}=\sum_{k=1}^{i} \mu^{k-1} S_{k}
$$

Since $\mu<1$ and $\left\|S_{i}\right\| \leq C$, we conclude that $S-S_{i}$ converges; therefore, $S_{i}$ converges.

Because the modified EMD sifting algorithm always converges, we can combine it with our second type of singular operator to decompose a signal into a superposition of narrow band signals. First, we use the modified EMD sifting algorithm to decompose a signal into a superposition of outputs, and then apply Algorithm II to obtain the local narrow band signals of each output. The following example demonstrates the efficiency of the proposed hybrid method.

Example 4: Individually, Algorithms I, II, and EMD can not correctly separate the following signal:

$$
S(t)=\sin (2 t)-(1+\cos (4 t)) / 4+\sin (8 t)-\cos (16 t) / 3 .
$$

The first two terms of the signal can not be separated by EMD or the first type of singular operator, while the third and the fourth terms can not be correctly decomposed by the first and second types of singular operator. However, the proposed hybrid method can decompose the signal correctly, as shown in Figure 4. 

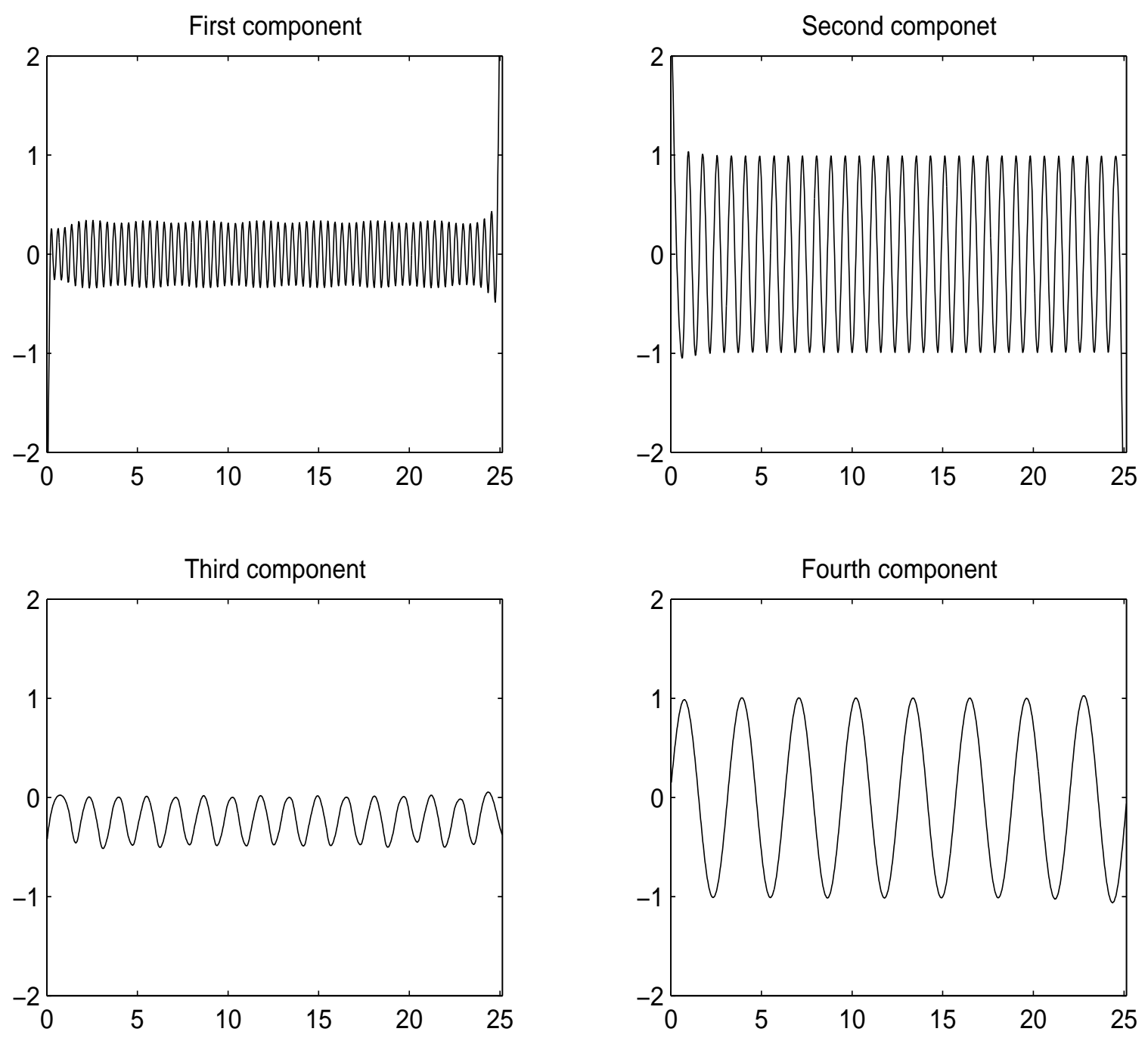

Figure 4: The decomposition results of our hybrid approach for the signal $\sin (2 t)-$ $\cos (4 t) / 4+\sin (8 t)-\cos (16 t) / 3$. The top left signal is $\cos (16 t) / 3$, the top right is $\sin (8 t)$, the bottom left is $-(1+\cos (4 t)) / 4$, and the bottom right is $\sin (2 t)$. Here, we set $\mu$ in the modified EMD sifting algorithm to 0.99, and $\lambda$ in Algorithm II to 0.025 . 


\section{Discussion and Application to Real-world Sig- nals}

The examples presented so far are constructed so that we can analyze and verify the solutions of different algorithms. We now describe the application of the proposed methods to two real-world signals, for which we do not have ground truths. Before applying our methods to the signals, we consider three issues related to the efficacy of the methods. First, we need to decide which decomposition method to use. We have introduced two types of singular local linear operators and a hybrid method, which combines a modified EMD algorithm and the second type singular operator. Using the first type of singular operator, we assume that the local average of a component signal is zero; while for the second type of singular operator, we assume that a component signal can be approximated as a local harmonic function. The class of signals of a zero local average is larger than the class of signals of the local harmonic components. For example, stochastic signals of the autoregressive moving average model are in the null space of the first type of singular operator, but the signals do not contain a local harmonic component. Because our objective it to extract the local narrow band components of a signal, we use either the second type of singular operator or the hybrid method. The second type of singular operator can be used effectively when the main frequencies of a signal are known, as shown in Figure 5. For all other cases, the hybrid method should be used because it combines the features of the EMD algorithm and the second type of singular operator. The modified EMD algorithm extracts the oscillatory components from a signal, then the second type of singular operator decomposes each oscillatory component into a superposition of local narrow band signals.

The second issue is that we need to determine the parameter $n$ in (6), which models the local harmonic component of a signal. Our experiment results show that signals can be separated properly by setting $n$ to 2 (modeling a neighborhood of $t$ as $(a t+b) \cos (\varpi t+c))$. We also need to determine the parameter $\lambda$ in $(21)$. At present, for all the examples, we choose the parameters based on the experiments. We are currently investigating other approaches, such as that proposed in [7], for estimating the parameter. The third issue relates to the complexity of our algorithm, which can be determined by inverting the matrices in (18) and (21). Because our operators are 
local, the matrices are band diagonal. According to [15, 10], the inverse of a band diagonal matrix can be solved with a complexity linear to the size of the signal. Thus, the proposed algorithms can be executed efficiently.
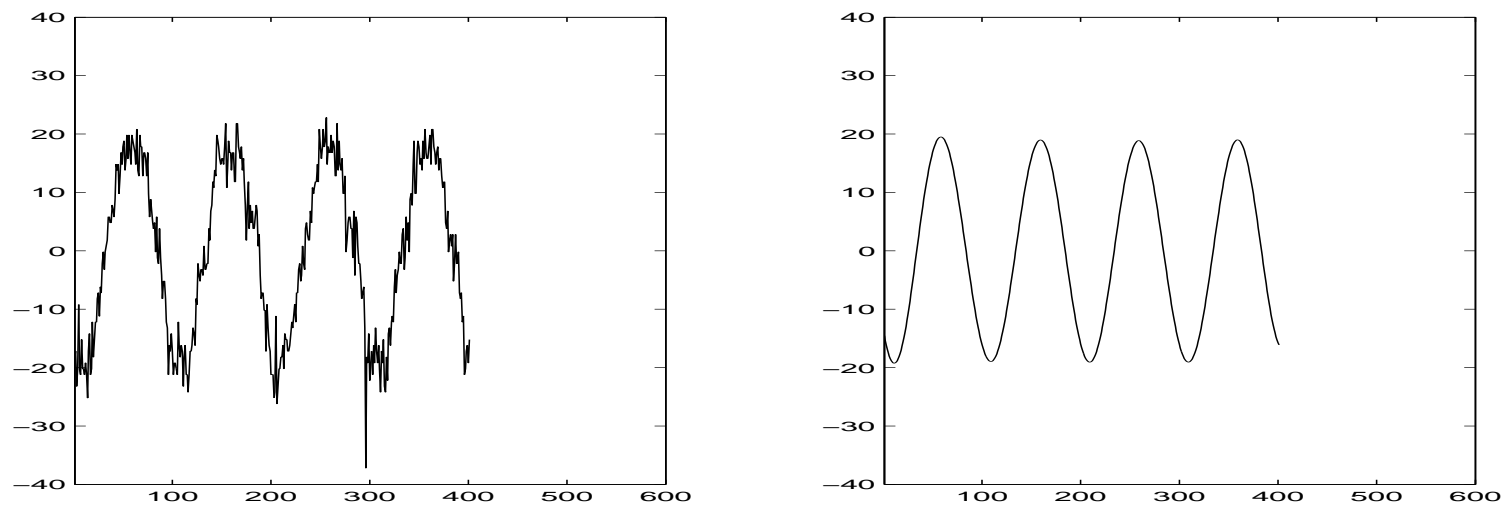

Figure 5: Left: A noisy signal from a gas detector. Right: The "clean" signal. The parameter $\lambda$ is set to 0.9 .

The left-hand subfigure of Figure 5 shows a noisy signal from a gas detector. The amplitude indicates the density of the gas, and the non-stationary noise is from the gas detector device. The main frequency of the signal was provided by the gas company. We thus substituted the frequency in the second type of singular operator to extract the "clean" signal, as shown in the right-hand subfigure of Figure 5.

Figure 6 shows the signal of Poland's daily electricity consumption from 1990 to 1994 [8]. Using the EMD algorithm, we obtain the first and most important IMF of the signal, as shown in Figure 7. The spectrum of the IMF, shown in Figure 8, indicates that the IMF contains three main frequency components at 200, 400, and 600 respectively. We use the proposed hybrid algorithm to decompose the signal into the three components, as shown in Figure 9. The spectrum of each component contains only one main frequency component. They are at 600, 400, and 200 in the spectra of the first, second, and third components respectively. Although, the three main frequency components obtained by EMD algorithm and our algorithm are match, the decompositions of the signal in Figure 6 by EMD and our algorithm are different. For EMD, all the main peaks are in the same IMF, while the peaks of our decomposition are in different components. This observation could lead to different explanations of the consumption rules of the signal, but we do not have sufficient knowledge about the signal to make an assessment. 


\section{Conclusion}

We propose an operator-based method for local narrow band signal decomposition that uses two singular local linear operators: an integral operator and a differential operator. Based on the operators, we develop two decomposition algorithms that extract either the first or the second type of local narrow band signal at each decomposition. We show that the EMD algorithm can be modified so that it always converges, but the solution is not necessarily an IMF. Because the solution of the modified EMD algorithm is a larger class of signals than the local narrow band signals, we propose a hybrid approach that combines the proposed method with the modified EMD algorithm to correctly decompose a signal into a superposition of local narrow band signals. In our future work, we will develop procedures to estimate the regularization parameter in our algorithms and make our operator estimation procedure more robust against the variations of local extremal points. It would also be interesting to derive the null spaces of the proposed operators and the relationships between the null spaces of different operators and the IMF space.

Acknowledgement: We would like to thank the anonymous reviewers for their insightful comments.

\section{References}

[1] B. Boashash, "Estimating and interpreting the instantaneous frequency of a signal. Part 1: fundamentals", Proc. IEEE, part I, vol. 80(4), pp. 520-538, 1992.

[2] B. Boashash, "Estimating and interpreting the instantaneous frequency of a signal. Part 2: algorithms and applications", Proc. IEEE, part II, vol. 80(4), pp. 540-568, 1992.

[3] A. Bovik, "Analysis of multichannel narrow-band filters for image texture segmentation," IEEE Transactions on Signal Processing, vol. 39(9), pp. 2025-2043, 1991.

[4] R. Carmona, W. L. Hwang, and B. Torresani, "Characterization of signals by the ridges of their wavelet transform," IEEE Trans. on Signal Processing, vol. 45, no. 10, pp. 2586-2590, 1997. 
[5] R. Carmona, W. L. Hwang, and B. Torresani, "Multiridge detection and timefrequency reconstruction," IEEE Trans. on Signal Processing, vol. 47, no. 2, pp. 480-492, 1999.

[6] P. Flandrin, G. Rilling, and P. Goncalves, "Empirical mode decomposition as a filter bank," IEEE Signal Processing Letters, vol. 11(2). pp. 112-114, 2004.

[7] G. Golub, M. Heath, and G. Wahba, "Generalized cross-validation as a method for choosing a good ridge parameter," Technometrics, vol. 21, pp. 215-223, 1979.

[8] A. C. Harvey and T. Trimbur, "General model-based filters for extracting cycles and trends in economic time series," The Review of Economics and Statistics, $85(2), 244-255$.

[9] N. E. Huang, Z. Shen, S. R. Long, M. L.Wu, H. H. Shih, Q. Zheng, N. C. Yen, C. C. Tung, and H. H. Liu, "The empirical mode decomposition and Hilbert spectrum for nonlinear and nonstationary time series analysis," Proc. R. Soc. London A, vol. 454, pp. 903V995, 1998.

[10] K. Lee and G. S. Chirikjian, "A New Perspective on O(n) Mass-Matrix Inversion for Serial Revolute Manipulators," IEEE International Conference on Robotics and Automation, 4722-4726, April 2005.

[11] Z. Liu and S. Peng, "Boundary Processing of bidimensional EMD using texture synthesis," IEEE Signal Processing Letters, vol. 12(1), pp. 33-36, 2005.

[12] T. Lu, L. Liang, and X. Wang, "Temporal and rate representations of timevarying signals in the auditory cortex of awake primates," Nature Neuroscience 4, pp. $1131-1138,2001$.

[13] R. J. Mcaulay and T. F. Quatieri, "Speech analysis/synthesis based on a sinusoidal representation," IEEE Trans. Acoust., Speech, Signal Processing, vol. ASSP-34, pp. 744-754, 1986.

[14] J. C. Nunes, Y. Bouaoune, E. Delechelle, et al., "Image analysis by bidimensional empirical mode decomposition," Image and Vision Computing, vol. 21(12), pp. 1019-1026, 2003. 
[15] W. H. Press, S. A. Teukolsky, W. T. Vetterling, and B. P. Flannery, "Numerical Recipes in FORTRAN 77: The Art of Scientific Computing," Cambridge University Press, 1992.

[16] D.S.G. Pollock. "Methodology for trend estimation," Economic Modelling, 18(1), 75-96, 2001.

[17] G. Rilling, P. Flandrin, "One or Two Frequencies? The Empirical Mode Decomposition Answers," preprint can be found http://prunel.ccsd.cnrs.fr/ensl00113834 .

[18] G. Rilling, P. Flandrin, and P. Gonealves, "On empirical mode decomposition and its algorithms," IEEE-EURASIP Workshop on Nonlinear Signal and Image Processing, NSIP-03, Grado (I), 2003.

[19] Z. Wu and N. E. Huang, "A study of the characteristics of white noise using the empirical mode decomposition method," Proc. R. Soc. London, Ser. A, vol. 460, pp. 1597-1611, 2004 


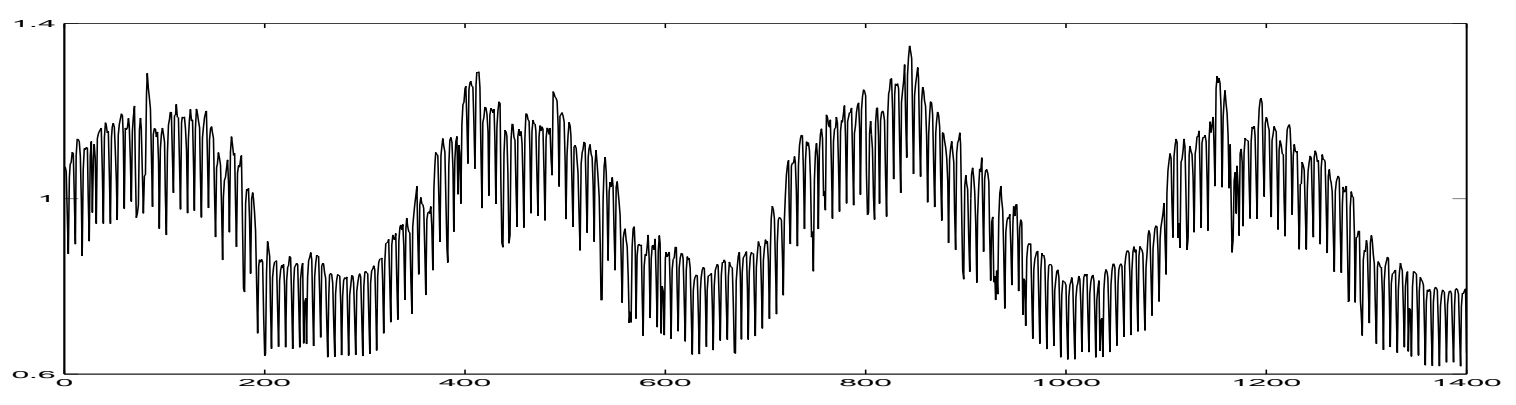

Figure 6: Signal of Poland's daily electricity consumption from 1990-1994.

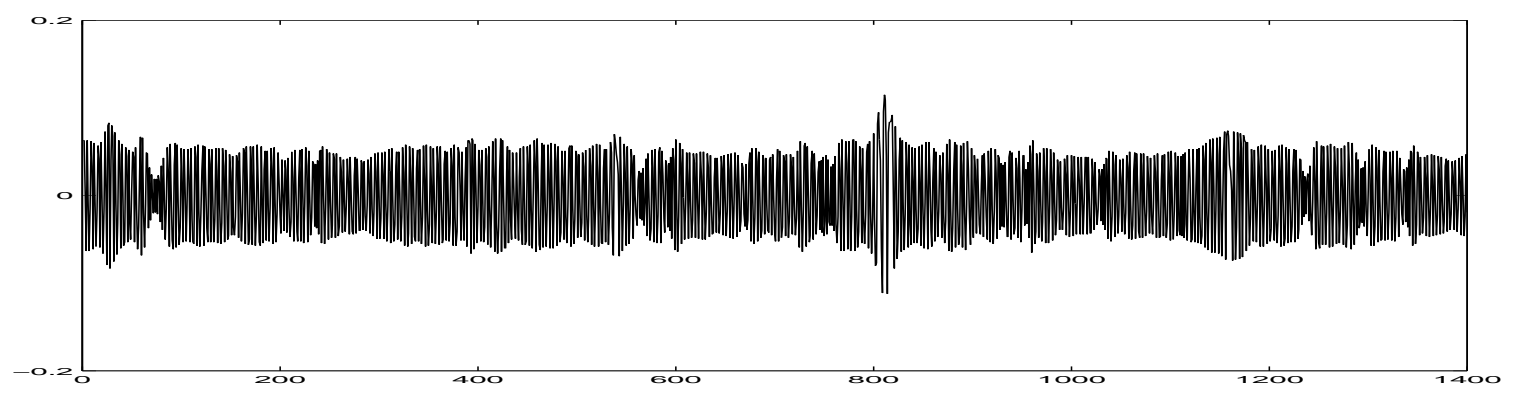

Figure 7: The first IMF obtained by the EMD algorithm for the signal in Figure 6. The IMF is an oscillatory component containing three main frequency peaks.

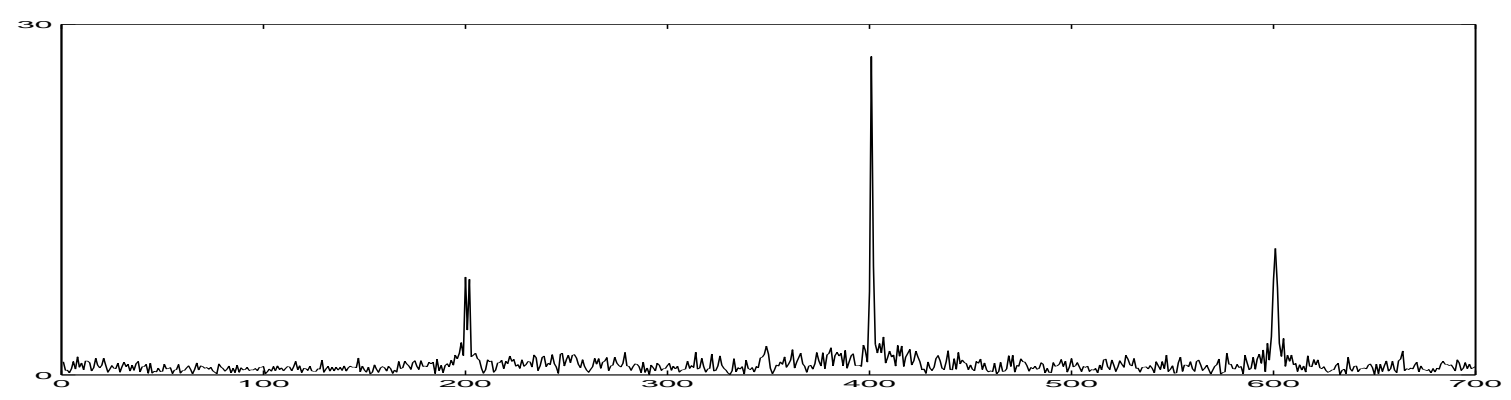

Figure 8: The spectrum showing the three main frequency peaks of the IMF in Figure 7. 

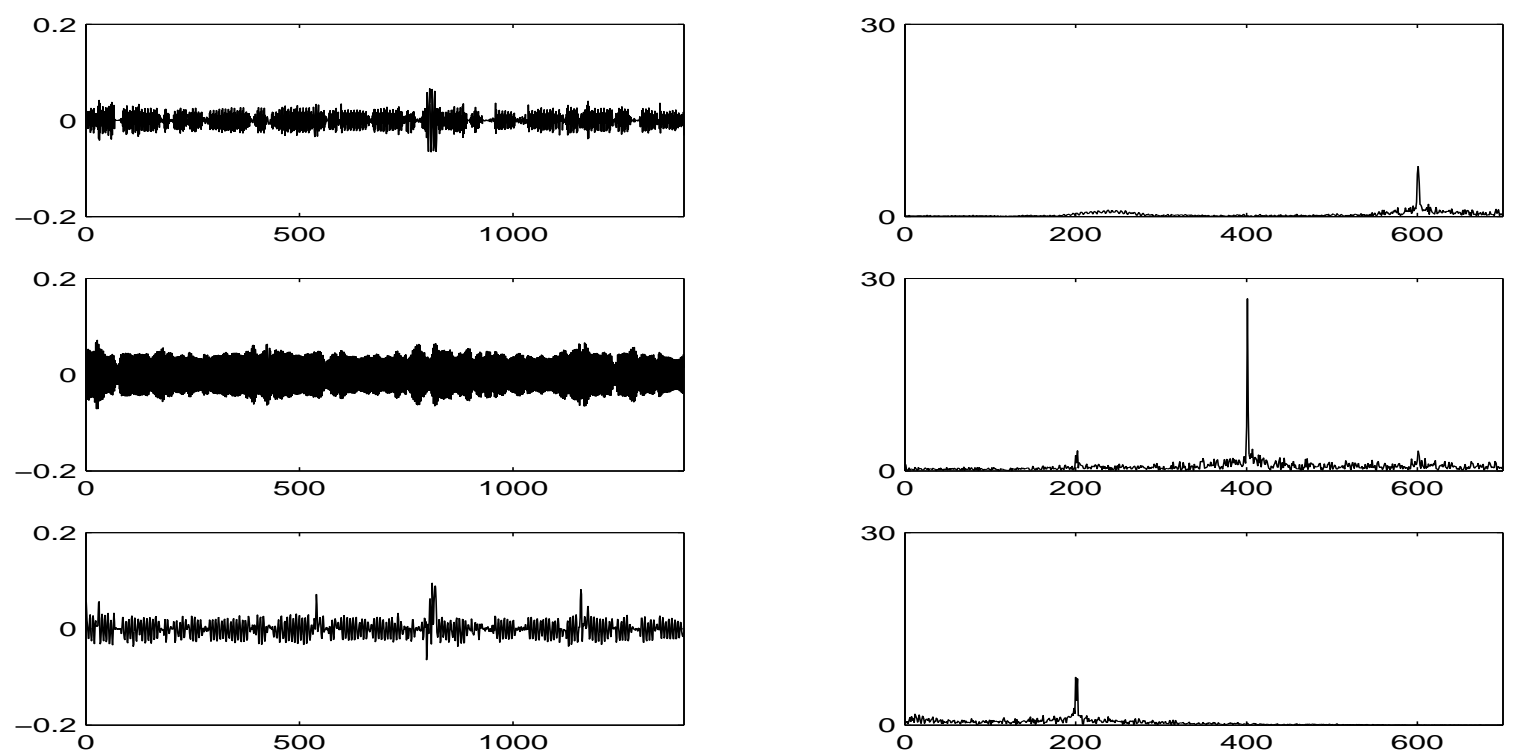

Figure 9: Three components extracted by the proposed hybrid method. The lefthand column shows the three components, and the right-hand column shows their respective spectrums. Each component has one main frequency peak. The parameter $\lambda$ is set to 0.005 when we use Algorithm II, and the parameter $\mu$ in the modified EMD sifting algorithm is set to 0.9. 